\title{
Relación entre el nivel de apoyo familiar en el cuidado y la calidad de vida de Ios pacientes con diabetes mellitus tipo 2, atendidos en la consulta externa del Hospital Nacional Guillermo Almenara Irigoyen, Lima, Perú
}

\author{
Relationship between the level of family support in the care and quality of life of patients with type \\ 2 diabetes mellitus treated at the outpatient clinic of the Guillermo Almenara Irigoyen National \\ Hospital, Lima, Peru
}

Gaby Sonia Chávez Zegarra'

\begin{abstract}
RESUMEN
Objetivo: Determinar la relación entre el nivel de apoyo familiar en el cuidado y la calidad de vida de los pacientes con Diabetes Mellitus tipo 2 atendidos en la Consulta Externa del Servicio de Endocrinología del HNGAI. Metodología: El estudio fue de diseño no experimental de tipo cuantitativo correlacional, de corte transversal empleando una muestra de 142 pacientes con diagnóstico de DM tipo 2. Para la recolección de datos se aplicaron dos instrumentos consolidados en un solo documento; para medir la calidad de vida de la persona se empleó el cuestionario de Salud SF - 36, y el cuestionario MOS de Apoyo Social percibido para medir el nivel de apoyo familiar. Los estadísticos aplicados fueron de tipo descriptivo y para establecer la correlación se empleó r-Pearson, considerando que las variables a correlacionar son de tipo categóricas. Resultados: Se encontró que la mayoría de los pacientes con Diabetes Mellitus tipo 2 presenta un nivel de calidad de vida de regular (35.2\%) a malo (30.28\%), presenta apoyo familiar de bueno $(35.9 \%)$ a excelente $(22.5 \%)$ y, por último, el nivel de calidad de vida según el nivel de apoyo familiar es bueno (51.3\%), por lo que se determina que el apoyo familiar influye positivamente en la calidad de vida del paciente con DM tipo 2. Conclusión: Existe relación entre la calidad de vida y el apoyo familiar, excepto en la dimensión actividad física y transmisión de la salud que no tienen relación con el apoyo familiar en los pacientes con Diabetes Mellitus tipo 2.
\end{abstract}

Palabras clave: Diabetes mellitus tipo 2, calidad de vida, apoyo familiar.

\section{SUMMARY}

Objective: To determine the relationship between the level of family support in the care and quality of life of patients with type 2 diabetes treated at the Outpatient Service of Endocrinology HNGAI. Methodology: The study was non-experimental quantitative correlational design of cross- cutting using a sample of 142 patients diagnosed with type 2 diabetes. To collect data consolidated two instruments were applied in a single document, to measure the quality of life of the individual SF Health Questionnaire was used - 36 and the MOS Social Support Questionnaire to measure perceived level of family support. Statistical applied were descriptive and to establish the correlation Pearson $r$ was used whereas correlate variables are categorical type. Results: We found that most patients with type 2 diabetes has a quality of life regularly $(35.2 \%)$ to poor $(30.28 \%)$, has good family support (35.9\%) to excellent (22.5\%), and finally the quality of life according to the level of family support is good $(51.3 \%)$, so it is determined that the family support positively influences the quality of life of patients with type 2 diabetes. Conclusion: There is a relationship between quality of life and family support, except in the physical dimension and transmission of health that are unrelated to family support in patients with Type 2 Diabetes Mellitus activity.

Keywords: Diabetes mellitus type 2, Quality of Life, Family Support.

\footnotetext{
${ }^{1}$ Enfermera Supervisora del Departamento de Enfermería, Hospital Base Guillermo Almenara Irigoyen. Red Asistencial Almenara (RAA) EsSalud, Lima. Perú.
} 


\section{INTRODUCCIÓN}

En la actualidad, las enfermedades crónicas no transmisibles han alcanzado proporciones epidémicas en las Américas y contribuyen substancialmente a la morbilidad y mortalidad general.

Según la Organización Mundial de la Salud (OMS), se ha observado una tendencia ascendente de la Diabetes Mellitus en los últimos tiempos: en 1985, no menos de 30 millones de personas la padecían, cifra que se elevó a 100 en 1994, y a 165 en el 2000 , con un pronóstico de 239 millones en el 2010 y 300 en el 2025.

La Diabetes Mellitus (DM) constituye un problema personal y de salud pública de enormes proporciones, siendo una de las principales causas de invalidez y muerte prematura en la mayoría de los países desarrollados y es motivo de un importante deterioro en la calidad de vida de las personas afectadas (La Fuente, 2003).

Entre las estrategias que la persona con diabetes lleva a cabo para vivir con la enfermedad, se encuentra la búsqueda de ayuda y apoyo no solo del personal sanitario, sino también - y de forma destacada - de las personas de su red social, sobre todo de su familia. Se considera que ese apoyo es capaz de compensar el efecto del estrés originado por la enfermedad.

El apoyo social y familiar son considerados como factores determinantes en las conductas de adherencia a los tratamientos para la diabetes. La interacción entre la familia y la tipología de la enfermedad pueden tener una influencia positiva o negativa sobre el curso del proceso crónico. Se debe destacar que la familia es la fuente principal de apoyo social con que cuenta el paciente crónico para afrontar con éxito los problemas ocasionados por la enfermedad, subrayando el papel del cuidador primario, que es quien aporta el máximo apoyo instrumental, afectivo y emocional (De la Revilla, 2003).

El estudio tiene como objetivo determinar la relación que existe entre el nivel de apoyo de la familia en el cuidado y la calidad de vida de los pacientes con Diabetes Mellitus Tipo 2 (DM2), atendidos en la consulta externa del Servicio de Endocrinología del HNGAI.

\section{MATERIAL Y MÉTODOS}

El estudio fue de tipo cuantitativo, de diseño no experimental y correlacional, de corte transversal porque los datos fueron tomados en un momento dado.
La población estuvo conformada por los pacientes con Diabetes Mellitus tipo II que asisten a consulta externa del Servicio de Endocrinología del HNGAI, que conforman un promedio de 450 pacientes atendidos por mes.

El tamaño de la muestra se determinó según fórmula con un nivel de confianza del $95 \%$ y con un error aleatorio del 5\%, conformada por 142 pacientes, seleccionados por el método probabilístico aleatorio simple, a quienes se les aplicó dos instrumentos, consolidado en un solo documento:

Cuestionario de Salud SF-36. (The Short-Form-36 Health Survey) validado por el Medical Outcomes Trust (1995), y en el presente estudio se usará la versión española 1.4 (1999), consta de 36 preguntas que miden nueve dimensiones del estado de salud: Actividad física, Rol físico, Dolor, Salud general, Vitalidad, Función social, Rol emocional, Salud mental, Nivel de trasmisión de la salud, detectando tanto estados positivos como negativos de la salud física y estado emocional.

Cuestionario MOS de Apoyo Social Percibido. Fue desarrollado por Sherbourne y Stewart (1991) del grupo el MOSS-SSS, the Medical Outcomes Study Social Support Survey. Está conformado por 20 preguntas que miden: Apoyo emocional, Ayuda material o instrumental, Relaciones sociales de ocio y distracción, Apoyo afectivo referido a expresiones de amor y cariño.

Para la recolección de datos se solicitó la autorización respectiva de la institución, se procedió a la aplicación del instrumento por el propio investigador a la muestra seleccionada. Se realizó una entrevista a través de un cuestionario y se llenó los datos obtenidos de los pacientes que esperaban ser atendidos en la consulta externa del servicio de Endocrinología en un período de tres meses (enero a marzo del 2011).

La información obtenida fue tabulada y procesada a través del software SPSS Estatictics v19 (Statistical Package Scieces Social) para Windows. Los estadísticos aplicados fueron de tipo descriptivo y para establecer las correlaciones se empleó r-Pearson, considerando que las variables a correlacionar son de tipo numérico. 
Relación entre el nivel de apoyo familiar en el cuidado y la calidad de vida de los pacientes con diabetes mellitus tipo 2, atendidos en la consulta externa del Hospital Nacional Guillermo Almenara Irigoyen, Lima, Perú

\section{RESULTADOS}

Tabla 1

Datos generales de los encuestados con Diabetes Mellitus Tipo 2 atendidos en consulta externa del Servicio de Endocrinología del HNGAI.

\begin{tabular}{|c|c|c|c|}
\hline \multirow{2}{*}{ Datos generales } & & \multicolumn{2}{|c|}{$(n=142)$} \\
\hline & & $\mathrm{n}$ & $\%$ \\
\hline \multirow{3}{*}{ Género } & Masculino & 67 & 47.2 \\
\hline & Femenino & 75 & 52.8 \\
\hline & Del menor hasta 60 & 22 & 15.5 \\
\hline \multirow{5}{*}{ Edad (años) } & De 60 a 70 & 55 & 38.7 \\
\hline & De 70 a 80 & 52 & 36.6 \\
\hline & De 80 hasta el mayor & 13 & 9.2 \\
\hline & Soltero & 14 & 9.9 \\
\hline & Casado & 88 & 62 \\
\hline \multirow[t]{4}{*}{ Estado Civil } & Viudo & 29 & 20.4 \\
\hline & Conviviente & 10 & 7 \\
\hline & Otro & 1 & 0.7 \\
\hline & Superior & 39 & 27.5 \\
\hline \multirow{5}{*}{ Grado de instrucción } & Secundaria & 52 & 36.6 \\
\hline & Primaria & 42 & 29.6 \\
\hline & No estudio & 9 & 6.3 \\
\hline & Obrero & 3 & 2.1 \\
\hline & Jubilado & 77 & 54.2 \\
\hline \multirow[t]{5}{*}{ Ocupación } & Empleado & 14 & 9.9 \\
\hline & Su casa & 36 & 25.4 \\
\hline & Independiente & 12 & 8.5 \\
\hline & Lima Norte & 48 & 38.8 \\
\hline & Lima Sur & 3 & 2.1 \\
\hline \multirow[t]{4}{*}{ Procedencia } & Lima Centro & 39 & 27.5 \\
\hline & Lima Este & 52 & 36.6 \\
\hline & Lima Oeste & 0 & 0 \\
\hline & Normal & 31 & 21.8 \\
\hline \multirow[t]{3}{*}{ Índice de masa corporal } & Sobrepeso & 65 & 45.8 \\
\hline & Obeso & 46 & 32.4 \\
\hline & $60-140 \mathrm{mg} / \mathrm{dl}$ & 67 & 47.2 \\
\hline \multirow[t]{3}{*}{ Niveles de glucosa } & $140-250 \mathrm{mg} / \mathrm{dl}$ & 63 & 44.4 \\
\hline & $250-500 \mathrm{mg} / \mathrm{dl}$ & 12 & 8.5 \\
\hline & Del menor hasta 5 & 33 & 23.2 \\
\hline \multirow{5}{*}{$\begin{array}{l}\text { Tiempo que padece la enfermedad } \\
\text { (años) }\end{array}$} & De $5-10$ & 44 & 31 \\
\hline & De $10-20$ & 42 & 29.6 \\
\hline & De $20-30$ & 12 & 8.5 \\
\hline & De 30 hasta el mayor & 11 & 7.7 \\
\hline & Del menor hasta 5 & 59 & 41.5 \\
\hline \multirow{4}{*}{$\begin{array}{l}\text { Tiempo de tratamiento de la } \\
\text { enfermedad (años) }\end{array}$} & De $5-10$ & 37 & 26.1 \\
\hline & De $10-20$ & 37 & 26.1 \\
\hline & De 20 a más. & 9 & 6.3 \\
\hline & 0 & 4 & 2.8 \\
\hline \multirow{3}{*}{$\begin{array}{l}\text { Número de familiares que brindan } \\
\text { apoyo }\end{array}$} & $1-2$ & 42 & 29.6 \\
\hline & $3-5$ & 71 & 50 \\
\hline & $6-8$ & 25 & 17.6 \\
\hline
\end{tabular}


La tabla 1 muestra que respecto al género de los encuestados, el $47.2 \%$ son de género masculino y el $52.8 \%$ de los mismos son de género femenino.

Según la edad, el $38.7 \%$ tienen entre 60 y 70 años, el $26.6 \%$ entre 70 y 80 años, solo el $15.5 \%$ son menores de 60 años y el 9.2 mayores de 80 años.

Respecto al estado civil, el $62 \%$ son casados por tanto viven acompañados de sus esposas/os, el $20.4 \%$ son viudos, el $9.9 \%$ son solteros y solo el $7 \%$ convivientes.

En cuanto al nivel de instrucción, el $36.6 \%$ tienen estudios secundarios, el $29.6 \%$ cuentan con instrucción primaria, el $27.5 \%$ tienen instrucción superior y solo el $6.3 \%$ son analfabetos.

En relación a la ocupación, el 54.2\% son jubilados, el $25.4 \%$ son amas de casa, lo que permite concluir que el $79.6 \%$ no trabajan, y solo el $20.4 \%$ trabajan como obreros empleados o en forma independiente.

Según la procedencia, el $38.8 \%$ son de Lima Norte, el $36.6 \%$ vienen de Lima Este y el $27.5 \%$ son de Lima Centro.
Según el índice de masa corporal, el $45.8 \%$ tienen sobrepeso, el $32,4 \%$ son obesos y solo el $21.8 \%$ tienen un índice de masa corporal normal.

Los resultados del nivel de glucosa en sangre revelan que el $47.2 \%$ tienen entre 60 y $140 \mathrm{mg} / \mathrm{dl}$ considerado como valores normales, el $44,4 \%$ tienen entre 140 y $250 \mathrm{mg} / \mathrm{dl}$ haciendo referencia a estado hiperosmolar y el $8.5 \%$ tienen más de $250 \mathrm{mg} / \mathrm{dl}$ compatibles con una cetoacidosis diabética.

En relación al tiempo que padecen la enfermedad, el $23.2 \%$ padecen esta enfermedad por menos de 5 años, el $31 \%$ entre 5 y 10 años, el $29.6 \%$ entre 10 y 20 años, y el $16.2 \%$ de 20 a más años.

Con respecto al tiempo de tratamiento, el 41.5\% reciben tratamiento desde hace menos de 5 años; el $26.1 \%$ entre 5 y 10 años, también el $26.1 \%$ entre 10 y 20 años y solo el $6.3 \%$ desde hace más de 20 años.

Asimismo respecto al número de familiares cercanos o amigos íntimos que apoyan a estos pacientes, el $79.6 \%$ tienen entre 1 y 5 personas, el $25 \%$, entre 6 y 8 personas y el $2.8 \%$ no tienen ningún familiar o amigos que lo apoyen, lo cual es preocupante si tenemos en cuenta que el mayor porcentaje de encuestados son adultos mayores.

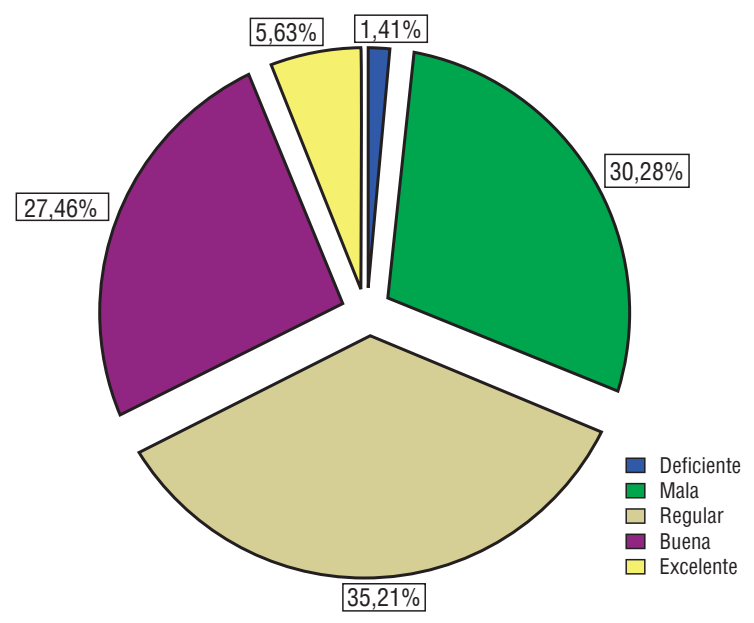

\section{Figura 1}

Nivel de Calidad de vida de los pacientes con Diabetes Mellitus Tipo 2 atendidos en consulta externa del Servicio de Endocrinología del HNGAI.

En relación a los hallazgos sobre la calidad de vida de los encuestados con Diabetes Mellitus tipo 2 atendidos en la Consulta Externa del Servicio de
Endocrinología del HNGAI, se encontró que el 35.2\% es regular, el $30.3 \%$ es mala, el $27.5 \%$ buena, el $5.6 \%$ excelente y solo el $1.4 \%$ deficiente. 


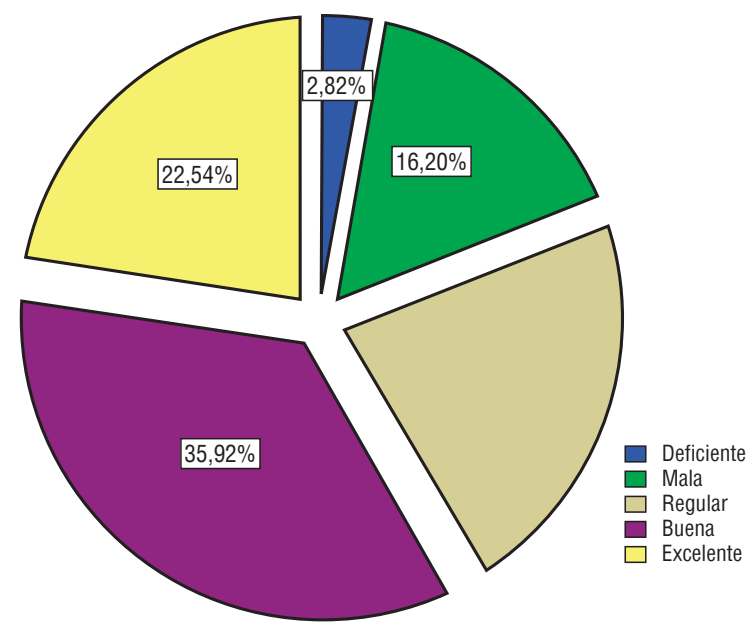

\section{Figura 2}

Nivel de Apoyo Familiar de los pacientes con Diabetes Mellitus Tipo 2 atendidos en consulta externa del Servicio de Endocrinología del HNGAI.

Este hallazgo refleja que el nivel de apoyo familiar desde una perspectiva funcional y estructural de los encuestados con diabetes Mellitus tipo 2 atendidos en la Consulta Externa del Servicio de Endocrinología del HNGAI, es bueno en el $35.9 \%$, es excelente en el $22.5 \%$, a diferencia del $22.5 \%$ que la considera regular, el $16.2 \%$ malo y solo el $2.8 \%$ es deficiente.

\section{Tabla 2}

Estadístico de relación de r-Pearson para las dimensiones de calidad de vida con nivel de apoyo familiar

\begin{tabular}{lll}
\hline & $\begin{array}{l}\text { R } \\
\text { (Pearson) }\end{array}$ & Sig. $^{*}$ \\
\hline Actividad física con nivel de apoyo familiar & 0.148 & 0,079 \\
Rol físico con nivel de apoyo familiar & 0.257 & 0.002 \\
Dolor corporal con nivel de apoyo familiar & 0.258 & 0.002 \\
Salud general con nivel de apoyo familiar & 0.345 & 0.000 \\
Vitalidad con nivel de apoyo familiar & 0.434 & 0.000 \\
Función social con nivel de apoyo familiar & 0.480 & 0.000 \\
Rol emocional con nivel de apoyo familiar & 0.456 & 0.000 \\
Salud mental con nivel de apoyo familiar & 0.382 & 0.000 \\
Transmisión de salud con nivel de apoyo familiar & 0.133 & 0.115 \\
\hline
\end{tabular}

$* \mathrm{p}>.05$.

En la tabla 2 se observa que en relación a la actividad física y los niveles de transmisión de la salud con el nivel de apoyo familiar no existe relación significativa $(p>$.05).
Por otro lado, existe relación significativa entre las demás dimensiones de calidad de vida con nivel de apoyo familiar: rol físico, dolor corporal, salud general, vitalidad, función social, rol emocional y salud mental $(p<.05)$. 


\section{DISCUSIÓN}

Como parte de los resultados generales de los pacientes que presentan Diabetes Mellitus tipo 2 que participaron en el estudio, destaca el hecho de que el $52.8 \%$ son de género femenino, lo que coincide con el aumento de la incidencia de Diabetes Mellitus en mujeres adultas. Desde esta perspectiva, se considera a la mujer como débil física y psicológicamente, lo que provoca una despreocupación hacia su persona haciéndola más vulnerable en situaciones de crisis o de tensión (Delongis, FolKman, Lazarus, 1998).

Por otro lado, la edad del $75.3 \%$ está entre los 60 a 80 años, y según la OMS el grupo etario mayor de 60 años es denominado como adultos mayores, los cuales están en proceso del envejecimiento, siendo una etapa de la vida en que la persona pierde progresivamente la capacidad de adaptación y la aptitud de reaccionar adecuadamente a los cambios.

Respecto al estado civil de la población encuestada, el $69 \%$ son casados y/o convivientes, por tanto viven acompañados de sus esposas/os, lo cual revela que tienen una compañía de apoyo cercano que quizá no esté dotado de las habilidades para cuidar y que son necesarias para este paciente, puesto que la persona que es el acompañante puede presentar problemas para enfrentar los cambios propios de la enfermedad de la pareja.

En relación al nivel de instrucción, se encontró que el 36.6\% tienen estudios secundarios, el 29.6\% tienen instrucción primaria, lo cual significa que en su mayoría tienen un nivel de instrucción básico, permitiéndoles conservar su autosuficiencia, su adaptación social, y desarrollo social, mantenerse informados, y que se sientan parte de la sociedad, con funciones y roles sociales.

Los hallazgos antes descritos coinciden con los hallazgos evidenciados por investigadores como Cárdenas, Pedraza y Lerma (2005), quienes encontraron en su investigación sobre calidad de vida del paciente diabético que predominó el género femenino con $58 \%$, con una edad promedió de 58 años, y con una escolaridad de cinco años cursados. También lo corroboran De los Ríos, Sánchez, Barrios y Guerrero (2003) quienes, en su investigación, concluyeron que los pacientes con DM2 tienen mayor riesgo de deterioro de la calidad de vida en comparación a aquellos pacientes con un nivel educativo bajo y edad mayor de 50 años.

Los autores anteriormente mencionados también indican que el tiempo promedio de la enfermedad de los pacientes sometidos a su estudio de investigación fue de 9 años, y el $80 \%$ con un control pobre de peso y control glicémico, lo que coincide con esta investigación, donde se obtuvo que el $31 \%$ padecen esta enfermedad por entre 5 y 10 años, el $45.8 \%$ presenta sobrepeso y el $32,4 \%$ son obesos.

En el estudio de investigación de Veliz (2005) en México, también se encontró que un considerable número de pacientes diabéticos presentaban obesidad y niveles altos de glicemia, coincidiendo con los resultados de este estudio, donde se obtuvo como resultado sobre los nivel es de glucosa en sangre, que el $44,4 \%$ tienen entre 140 y $250 \mathrm{mg} / \mathrm{dl}$, lo cual hace referencia a una descompensación denominada: Síndrome Hiperglucémico o Hiperosmolar No Cetósico.

Otro dato general que debe ser mencionado es que el $8.5 \%$ de los pacientes encuestados presentan más de $250 \mathrm{mg} / \mathrm{dl}$ de glucosa sanguínea.

En cuanto al número de familiares cercanos o amigos íntimos que apoyan a los pacientes con diabetes Mellitus tipo 2, se encontró que el $32.4 \%$ están entre 0 y 2 personas, y el $50 \%$ entre 3 y 5 personas. Este dato es muy significativo, si tenemos en cuenta que la familia, los amigos y vecinos son una fuente importante de apoyo de los adultos mayores. Este hallazgo guarda relación con lo que Rodríguez y Guerrero (1997) encontraron en su investigación sobre la importancia del apoyo familiar en el control de la glucemia, cuyo resultado muestra que el apoyo familiar que recibe el paciente se asocia significativamente a la presencia de glucemia $<140 \mathrm{mg} / \mathrm{dl}$.

En los resultados específicos del presente estudio, se encontró que la calidad de vida de los encuestados con Diabetes Mellitus tipo 2, es regular en un 35.2\%, en el $30.3 \%$ es mala, en el $27.5 \%$ buena, en el $5.6 \%$ excelente y solo en el $1.4 \%$ es deficiente, deduciendo que el nivel de calidad de vida del mayor porcentaje de los encuestados está en un nivel entre regular y malo. Estos resultados permiten afirmar que la calidad de vida de estos pacientes diabéticos no solo necesita integrarse a un régimen de tratamiento y vivir con él, sino que también están expuestos a la posibilidad de presentar complicaciones agudas o crónicas de la enfermedad como la hipoglicemia, hiperglicemia, pie diabético, retinopatía, entre otros, los cuales tienen un impacto significativo en muchos aspectos de su vida, como el trabajo, las relaciones interpersonales, el funcionamiento social y el bienestar físico y emocional.

Estos resultados muestran, como lo menciona Schumaker y Naughton (1996), que la calidad de vida 
en estos pacientes está representada por la percepción subjetiva, influenciada por el estado de salud actual, y la capacidad para realizar aquellas actividades importantes para el individuo.

Estas evidencias demuestran que, a medida que la enfermedad progresa, ocurren ajustes internos para preservar la satisfacción que la persona siente con la vida, por lo que podemos encontrar personas con grados importantes de limitación física que consideren que su calidad de vida es buena, lo cual coincide con lo mencionado por Leplége y Hunt, (1998).

Los hallazgos sobre el nivel de apoyo familiar desde una perspectiva funcional y estructural de los encuestados con Diabetes Mellitus tipo 2, mostraron (Gráfico 2) que en el 35.9\% era bueno y en el $22.5 \%$ excelente; a diferencia del $22.5 \%$ que lo consideraba regular, el $16.2 \%$ malo y solo el $2.8 \%$ deficiente. Esto permite evidenciar que la mayoría de los pacientes sí perciben el apoyo familiar, posibilitando que estas personas mejoren su calidad de vida, ya que para este propósito, según Hidalgo (2005), el cumplimiento del rol de la familia mediante el apoyo, permite afianzar el autocuidado; asimismo estos resultados coinciden con los obtenidos por Magaña, Ayala y Benavides (2002) en su investigación sobre los hábitos de cuidado para la salud que desarrollan los familiares de los pacientes con Diabetes Mellitus donde concluyó que estos están encaminados a la prevención.

Los resultados antes descritos demuestran que el apoyo familiar es significativo y fundamental en la percepción de estos pacientes, considerando que el tipo de apoyo emocional e instrumental es necesario en el curso del desarrollo de la enfermedad. El apoyo familiar es particularmente importante en el paciente diabético, ya que esta enfermedad tiene un carácter de cronicidad, lo cual impacta en la familia. Se puede deducir que a mayor vinculación, mejor es el estado emocional de la persona, lo que permite que el sistema inmunológico no se deprima, previniendo complicaciones; en cambio, quienes perciben menor apoyo familiar están predispuestos a experimentar trastornos emocionales y físicos, como lo afirman Portilla, Romero y Román, (1991).

Para concluir el análisis sobre el nivel de calidad de vida según el nivel de apoyo familiar que presentan los pacientes con Diabetes Mellitus tipo 2 atendidos en consulta externa del Servicio de Endocrinología del HNGAI, se determina que del 100\% (2) que tiene una deficiente calidad de vida, el nivel de apoyo familiar es también deficiente o malo, así mismo del 100\% (43) que tienen una mala calidad de vida el nivel, de apoyo familiar es entre malo y deficiente en un $51.2 \%$ (22); pero llama la atención que haya un $44.2 \%$ (19) que presentan un nivel de buen a excelente apoyo familiar. De igual modo, los que tienen regular calidad de vida presentan de deficiente a regular apoyo familiar en un $46 \%$ (23), así también causa suspicacia que el 54\% (27) presentan un apoyo familiar entre bueno y excelente, asimismo sucede que del 100\% (39) que presentan buena calidad de vida, el $74.4 \%$ (29) representa de bueno a excelente nivel de apoyo familiar, en cambio el $25.7 \%$ (10) refieren tener malo o regular apoyo familiar. En lo que se refiere al nivel excelente de calidad de vida, se encontró que del 100\% (8) tienen entre bueno y excelente apoyo familiar. De los resultados obtenidos, se puede determinar que a mejor calidad de vida que presentan los encuestados, tienen mayor apoyo familiar; sin embargo, es preocupante que también haya una relación inversa en otros casos donde a menor calidad de vida hay mayor apoyo familiar, o a mayor calidad de vida haya menor apoyo familiar.

Los resultados antes descritos evidencian que a pesar que su nivel de calidad de vida es malo o regular, ellos perciben que el nivel de apoyo familiar es de bueno a excelente en mayor porcentaje; a diferencia de los que su nivel de calidad de vida es de bueno a excelente, perciben que el nivel de apoyo familiar es de bueno a excelente en mayor porcentaje, lo que nos permite deducir que no hay una relación directa entre el nivel de calidad de vida y el nivel de apoyo familiar.

Por otro lado, la mayoría de expertos ven a la calidad de vida como una estructura multidimensional, sin embargo, es claro que la calidad de vida es una noción eminentemente humana que se relaciona con el grado de satisfacción que tiene la persona, con su situación física, su estado emocional, su vida familiar, amorosa, social, así como el sentido que le atribuye a su vida, entre otras cosas.

En conclusión, con respecto al nivel de apoyo familiar en el cuidado se encontró que la mayoría de los pacientes con Diabetes Mellitus tipo 2 lo perciben de bueno a excelente. En relación a la calidad de vida de los pacientes con Diabetes Mellitus tipo 2, la mayoría lo percibe de malo a regular. Asimismo, se encontró que existe relación entre las dimensiones de calidad de vida y apoyo familiar, excepto en la dimensión actividad física y transmisión de la salud que no tienen relación con el apoyo familiar en los pacientes con Diabetes Mellitus tipo 2. 


\section{Declaración de financiamiento y de conflicto de intereses:}

El estudio fue financiado por la autora, quien declara no tener algún tipo de conflicto de interés en la investigación realizada.

\section{Correspondencia:}

Mg. Gaby Sonia Chávez Zegarra

Hospital Base Guillermo Almenara Irigoyen

Red Asistencial Almenara (RAA) - EsSalud

Av. Grau 800, La Victoria, Lima, Perú

Correo electrónico: gchavezz@hotmail.com

\section{REFERENCIAS BIBLIOGRÁFICAS}

Armendáriz Lorente, (2002). "Un diabético en la familia". Extraído de:controlatudiabetes.com/un_diabetico_en la familia.htm-26k.

Cabrera E, Suárez L, Díaz O (2000). Nuevos criterios para clasificar la diabetes mellitus. Rev. Cubana Endocrinológica; 11 (1): 515.

Cárdenas V, Pedraza C, Lerma R (2005). Calidad de vida del paciente con Diabetes Mellitus tipo 2. Revista de la Universidad Autónoma de Nuevo León Monterrey; México, Ciencia UANL. Julio-Septiembre, (3): 351357.

De los Ríos José, Sánchez Juan, Barrios Pedro y Guerrero Verónica (2003). "Calidad de vida en pacientes con diabetes mellitus tipo 2".

Enciclopedia libre Universal en Español (2006). Calidad de vida. Extraído de http://enciclopedia.us.es/index. php/Calidad_de_vida
La Fuente Robles N, Cruz Arándiga R, Granados Alba A, Batres Sicilia JP, Castilla Romero ML. (2003). Guía de atención enfermera a personas con diabetes. Granada. Asociación Andaluza de Enfermería Comunitaria.

López Manuel y Col. (2006). Adaptación y validación del instrumento de calidad de vida Diabetes 39 en pacientes mexicanos con diabetes mellitus tipo 2. México. pp. 201- 207.

Peña V. y Padilla M. (1997). "La familia y la comunidad como red de soporte social”. Lima. CEDRO.

Sociedad Andaluza de Medicina Familiar y Comunitaria. (2010). Grupo de trabajo sobre diabetes. Guía Clínica de Diabetes para Atención Primaria.

Recibido: $22 / 04 / 2013$

Aceptado: 24/09/2013 